\title{
Doppler coherence imaging and tomography of flows in tokamak plasmas (invited) ${ }^{\text {a) }}$
}

\author{
J. Howard, ${ }^{1, b)}$ A. Diallo, ${ }^{1, c)}$ M. Creese, ${ }^{1}$ B. D. Blackwell, ${ }^{1}$ S. L. Allen, ${ }^{2}$ R. M. Ellis, ${ }^{2}$ \\ G. D. Porter, ${ }^{2}$ W. Meyer, ${ }^{2}$ M. E. Fenstermacher, ${ }^{2}$ N. H. Brooks, ${ }^{3}$ M. E. Van Zeeland, ${ }^{3}$ \\ and R. L. Boivin ${ }^{3}$ \\ ${ }^{1}$ Plasma Research Laboratory, The Australian National University, Canberra 0200, Australia \\ ${ }^{2}$ Lawrence Livermore National Laboratory, Livermore, California 92093, USA \\ ${ }^{3}$ General Atomics, P.O. Box 85608, San Diego, California 92186-9784, USA
}

(Presented 20 May 2010; received 21 June 2010; accepted 18 August 2010; published online 25 October 2010)

\begin{abstract}
This article describes the results of spatial heterodyne Doppler "coherence imaging" of carbon ion flows in the divertor region of the DIII-D tokamak. Spatially encoded interferometric projections of doubly ionized carbon emission at $465 \mathrm{~nm}$ have been demodulated and tomographically inverted to obtain the spatial distribution of the carbon ion parallel flow and emissivity. The operating principles of the new instruments are described, and the link between measured properties and line integrals of the flow field are established. An iterative simultaneous arithmetic reconstruction procedure is applied to invert the interferometric phase shift projections, and the reconstructed parallel flow field amplitudes are found to be in reasonable agreement with UEDGE modeling. () 2010 American Institute of Physics. [doi:10.1063/1.3492422]
\end{abstract}

\section{INTRODUCTION}

Doppler spectroscopy provides a wealth of information on the physics of plasma confinement in tokamaks. The standard approach makes use of a grating spectrometer that disperses the spectrum in one spatial dimension and reserves the other to obtain a 1D spatial section of the region of interest. ${ }^{1}$ In many cases, however, such as scrape-off-layer (SOL) and divertor spectroscopy, a 2D imaging capability is required in order to correctly interpret the spectral information. The power of both active and passive 2D optical imaging systems to reveal new physics has been demonstrated recently by a number of authors. ${ }^{2,3}$

Recently, we have shown that wide-field-of-view "coherence-imaging" (CI) systems (polarization interferometers) have the unique capability to obtain time-resolved 2D projections (images) of key spectral quantities in passive Doppler spectroscopy. ${ }^{4,5}$ In its simplest form, a polarization interferometer consists of a birefringent delay plate of suitable thickness sandwiched between crossed or parallel polarizers, combined with an optical interference prefilter to isolate the spectral features of interest. Because there are no slits, polarization interferometers allow for 2D spectral imaging using robust components with high optical throughput. The coherence information can be encoded on one or more spatial heterodyne carriers imprinted on the image ${ }^{6}$ to allow single-frame "snapshot" Doppler imaging.

The key elements in these systems are various forms of

\footnotetext{
a) Invited paper, published as part of the Proceedings of the 18th Topical Conference on High-Temperature Plasma Diagnostics, Wildwood, New Jersey, May 2010.

${ }^{b)}$ Electronic mail: john.howard@anu.edu.au.

${ }^{c}$ Present address: Princeton Plasma Physics Laboratory, Princeton, New Jersey, USA.
}

birefringent Savart plates which produce an angle-dependent interferometric phase shear to generate approximately parallel spatial interference fringes in the image plane. ${ }^{6,7}$ The interferogram can be demodulated and the local fringe amplitude and phase compared with the properties of a set of reference fringes. The instrument shares some common features with spatial heterodyne spectro-polarimetry for motional Stark effect imaging - a new polarimetric imaging modality recently tested in experiments on the TEXTOR tokamak. ${ }^{8,9}$

We have recently deployed snapshot systems for Doppler imaging the emission from the CII $514 \mathrm{~nm}$ and CIII $465 \mathrm{~nm}$ multiplets originating in the divertor and SOL regions of the DIII-D tokamak. ${ }^{5}$ Carbon ion flow measurements based on multiple discrete channel visible Doppler spectroscopy have also been reported previously. ${ }^{10,11} \mathrm{Be}-$ cause of the uncertainty in brightness-weighting, sophisticated spectrum-fitting routines that take account of Zeeman effect broadening must be employed to extract the flow fields, and the resulting flow estimates and their localization can have large uncertainties.

The major advantage of the CI approach to Doppler spectroscopy of inhomogeneous media is the direct link between interferometric properties and well-defined integrals of plasma brightness, flow velocity, and temperature. ${ }^{12}$ Subject to reasonable symmetry constraints, this then allows the tomographic unfolding of plasma flows and temperatures from 2D projections, obviating many of the otherwise difficult interpretation issues noted above. In this paper we present first results of tomographic unfolding of the emissivity and parallel flow speed of $\mathrm{C} 2+$ ions in the DIII-D divertor, and compare with UEDGE modeling ${ }^{13}$ of these quantities. 


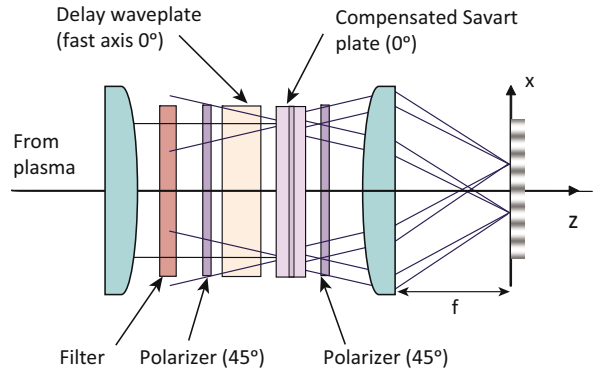

FIG. 1. (Color online) Optical arrangement for producing spatial heterodyne images of a Doppler broadened spectral line. The delay plate at $0^{\circ}$ resolves the polarized input into mutually delayed vertically and horizontally polarized components. These are spatially separated vertically using a zero-netdelay compensated Savart plate. The separation gives an angular shear in the phase shift between orthogonal polarization components which, when imaged through an analyzer at $45^{\circ}$, produces horizontally aligned interference fringes.

\section{OPTICAL SYSTEM AND INSTRUMENT RESPONSE}

While the optical system design and instrument response have been discussed in greater detail elsewhere, ${ }^{5}$ we here summarize the key system features as they pertain to imaging measurements of the $\mathrm{C} 2+$ multiplet at $465 \mathrm{~nm}$ in the region of the DIII-D divertor.

A tangentially viewing light collection system produces a real image of the lower divertor at the view port exit which is transferred by an imaging fiber cable to the interferometer and camera. Figure 1 shows a schematic layout of the interferometer. The radiation is propagating in the $z$ direction and the $x$-axis is taken to be vertical with the $y$-axis out of the page. The interferometer is bracketed by F-mount lenses of focal length 55 and $50 \mathrm{~mm}$ which collimate the light and produce a demagnified image of the optical fiber array onto the PCO Sensicam QE camera detector array $(8.9 \mathrm{~mm}$ $\times 6.7 \mathrm{~mm}$ ).

A first polarizer oriented at $45^{\circ}$ in the $x-y$ plane is followed by a $4 \mathrm{~mm}$ thick $\mathrm{LiNbO}_{3}$ waveplate with vertical fast axis $\left(0^{\circ}\right)$. The delay plate mutually delays polarization components parallel and perpendicular to the fast axis to produce a phase difference $\phi_{0}=2 \pi \nu_{0} \tau_{0}$ where $\nu_{0}$ is the radiation optical frequency and $\tau_{0}=L B / c$ is interferometric time delay, $L$ is the plate thickness, and $B$ its birefringence (see the Appendix).

A $4 \mathrm{~mm}$ thick $\mathrm{LiNbO}_{3}$ compensated Savart plate ${ }^{6,7}$ vertically separates the fast and slow polarization components to produce an angle-dependent shear in the phase difference. When imaged through the final analyzer oriented at $45^{\circ}$, this shear generates horizontally aligned interference fringes in the final lens focal plane. The phase difference at vertical position $x$ from the origin in the focal plane depends on the Savart plate thickness (beam separation $d$ ) and the lens focal length $f$ according to $\phi_{S}=2 \pi \nu_{0} \tau_{S}(x)$ where $\tau_{S}(x)=(d / c) x / f$.

The total interferometric phase therefore consists of contributions from the delay plate and Savart shearing plate such that the resulting interferogram is given by

$$
S_{ \pm}=\frac{I_{0}}{2}\left[1+\zeta \cos \left(\phi_{0}+\phi_{S}+\phi_{D}\right)\right]
$$

where $I_{0}$ is the emission brightness, $\zeta$ is the fringe contrast, and $\phi_{D}$, the phase shift produced by a Doppler shift of the

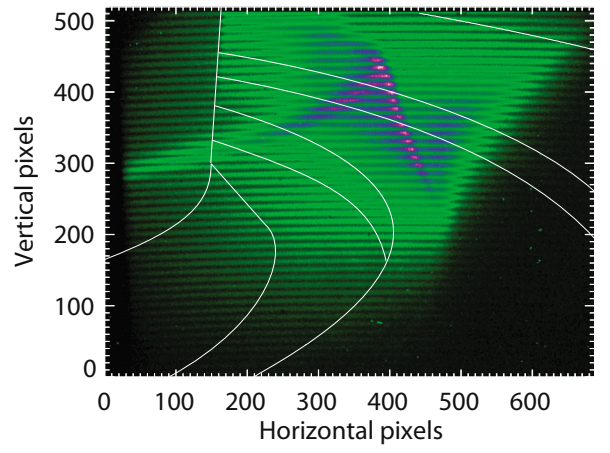

FIG. 2. (Color online) False color raw interferometric image for discharge no. 141170 at time of $3860 \mathrm{~ms}$ into the discharge. The wire-frame lines superimposed on the fringes show outlines of the divertor floor structure and the inside wall of the tokamak.

spectral line center, is proportional to the delay plate phase offset $\phi_{0}$.

The fringe contrast and phase depend on the properties of the spectrum in the optical passband. Analysis of spectra provided by the high resolution multichordal divertor spectrometer system indicates that the $465 \mathrm{~nm}$ line represents more than $95 \%$ of the radiated power in the passband, and that the relative brightness of the multiplet components does not appear to change with plasma conditions. The effects of the angle-dependent passband shift across the field-of-view are also found to be negligible. ${ }^{5}$

Based on the measured spectrum, we have modeled the variation of the fringe contrast with optical group delay and ion temperature. At the chosen lithium niobate delay plate thickness of $4 \mathrm{~mm}$ (approximately 1300 waves), the variation in fringe contrast over the expected range of ion temperatures $(10-20 \mathrm{eV})$ is only a few percent. In this case, the instrument is not suited for measuring the ion temperature, but is sensitive to flow speeds in the Mach number range of $0.1-1$ (at $10 \mathrm{eV}$ ) which produce phase shifts from 0.035 to $0.35 \mathrm{rad}$

\section{IMAGE PROCESSING AND DEMODULATION}

Figure 2 shows a typical $2 \times 2$ binned image of the CIII emission in the DIII-D divertor. A wire-frame overlay shows the outline of the divertor structure and inner wall of the vessel. Required exposure times are typically between 10 and $100 \mathrm{~ms}$ depending on the fiber transmission and plasma conditions. Carbon ion flow-induced distortion of the fringe phase fronts is evident on close inspection of the otherwise approximately horizontal fringe pattern. Various portions of the image field are not illuminated by the plasma and there is evidence of vignetting in the outer regions of the image. It should be noted that the interior wall of the tokamak is clad in carbon tiles and that, as a result, back reflections are not an important issue in this work. While these shortcomings affect the fidelity of tomographic reconstructions, they do not significantly change the structure of the inverted flow field.

A column-by-column wavelet-based demodulation procedure is used to extract the fringe properties. A three point median filter is usually applied to suppress radiation-induced noise and the brightness projection [see Eq. (A2)] is recovered by boxcar smoothing using a window of width equal to 

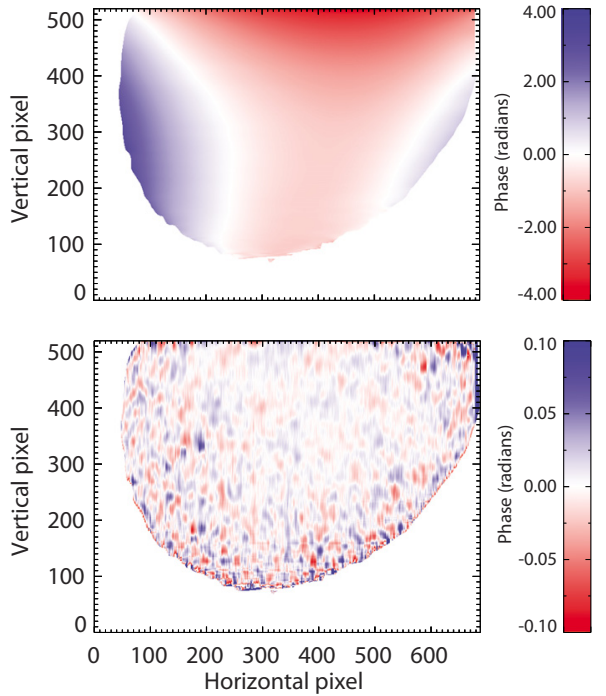

FIG. 3. (Color online) Top: Fitted reference phase image and bottom: the residual noise.

the fringe period (22 pixels in this case). The carrier fringes are extracted by factoring out the dc brightness and a fourth order Morlet wavelet-based demodulation algorithm is applied to obtain the fringe phase. The processing is usually restricted to regions where pixels exceed a photoelectron count of ten.

A suitable narrow spectral line source that falls within the interference filter passband was unavailable for calibrating these experiments. A reference phase image was constructed by using the first frame from the plasma image sequence (normally quite faint) and averaging over a set of discharges. In practice it is found that these images are generally featureless, and apart form a constant offset, provide a consistent and reliable phase reference. Nevertheless, there remains a fixed phase-noise pattern of rms value up to $0.1 \mathrm{rad}$, depending on image brightness. This noise is removed by using an eighth degree line-by-line polynomial fit to the reference phase image. The fitted image and residual phase noise are shown in Fig. 3. The linear carrier phase ramp has been removed to reveal the underlying hyperbolic fringe pattern associated with an uncompensated birefringent Y-cut delay plate.

The demodulated brightness and phase projections at representative times in a discharge (no. 141170) during which the plasma detaches from the divertor floor are shown in Fig. 4. The striations in the phase images are artifacts of the demodulation procedure and are due to the fact that the plasma brightness projection is changing on a spatial scale comparable with the fringe period. (Increasing the fringe spatial frequency would help reduce this effect.)

The phase image appears to be consistent with emissivity-weighted flows in opposing directions (see also Sec. V). By changing the delay plate thickness, it has been confirmed experimentally that the observed phase shifts scale in proportion to the optical delay offset $\phi_{0}$ [see also Eq. (2)]. In accordance with UEDGE modeling expectations, it is also found experimentally that reversing the sense of the toroidal field reverses the sense of the observed phase shift structures. Finally, the amplitude of the phase shift excursions are consistent with the near sonic flow speeds predicted by UEDGE. We therefore have confidence that the phase shift image truly represents the emissivity-weighted projection of the local flow velocity vector component in the direction of view.

\section{TOMOGRAPHIC TECHNIQUES}

As shown in the Appendix, for flows $v_{D}$ approaching the thermal speed that are often encountered in the SOL and divertor, the phase of the complex coherence gives an emissivity-weighted line integral of the fluid flow velocity component along the line-of-sight

$$
\frac{\phi_{D}}{\hat{\phi}_{0}} \approx \frac{1}{I_{0} c} \int_{L} e(\boldsymbol{r}) \boldsymbol{v}_{D} \cdot d \boldsymbol{l}
$$

where $I_{0}$ is the line-integrated emissivity $e(\boldsymbol{r})$.

In order to tomographically invert the phase projection, we assume toroidal symmetry so that a single $2 \mathrm{D}$ projection is sufficient to reconstruct scalar functions of radius $r$ and
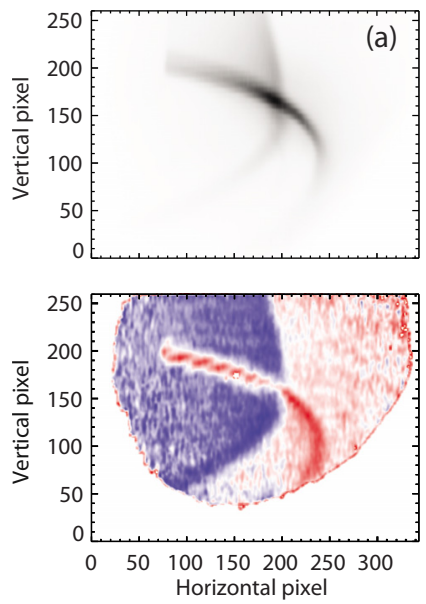
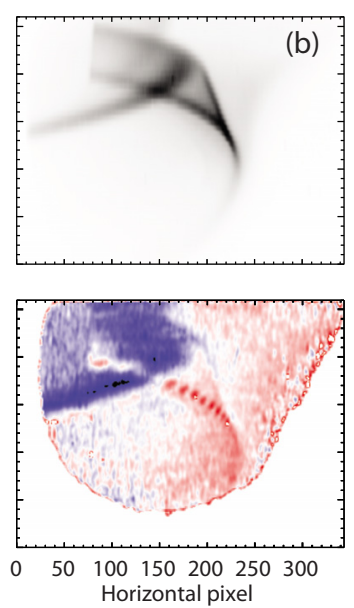
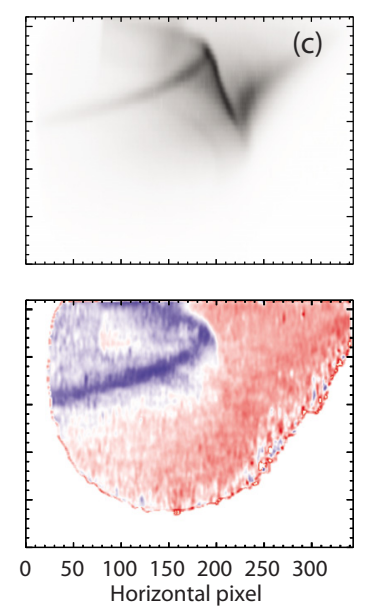
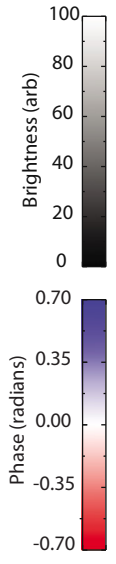

FIG. 4. (Color online) Demodulated brightness (top) and phase (bottom) projections at representative times during the divertor evolution for DIII-D discharge no. 141170: (a) $500 \mathrm{~ms}$, (b) $2000 \mathrm{~ms}$, and (c) $4000 \mathrm{~ms}$. The palette for the brightness images is inverted. See text for discussion. 


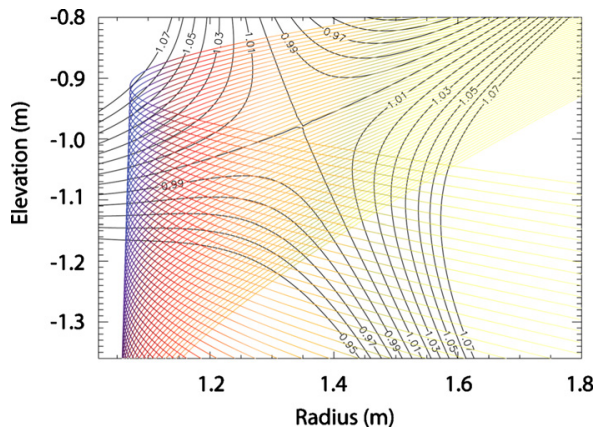

FIG. 5. (Color online) Typical $r-\mathrm{z}$ trajectories for an image column superimposed on the flux-surface structure in the divertor region. The trajectories are color-coded according to their weight in the system response matrix, with greatest weight near the tangency radius.

elevation $z$. We also assume that the flow is predominantly parallel to the magnetic field $\boldsymbol{v}_{D}=v_{D} \hat{\boldsymbol{B}}$. With this latter assumption, which is well supported by UEDGE modeling, the integral weighting factor $\hat{\boldsymbol{B}} . \hat{\boldsymbol{l}}$ can be calculated given knowledge of the viewing geometry and the magnetic equilibrium. With the local emissivity obtained by inverting the brightness projection $I_{0}$, it is possible to calculate the integral weight $I_{0}(r, z) \hat{\boldsymbol{B}} . \hat{\boldsymbol{l}}$ and so to reconstruct the scalar field $v_{D}(r, z)$ from the measurement $I_{0} \phi_{D}$ [see Eq. (2)].

The viewing geometry is estimated by matching vessel features occasionally observed in reflected plasma light with a 3D raytrace model of the internal structure. A $1 \mathrm{~cm}$ square Cartesian grid discretizes the reconstruction domain and each projection pixel is approximated as a simple line-of-sight integral over this grid (ignoring collection solid angle refinements, vignetting corrections, or possible internal reflections). A "response" matrix $R_{i j}$ is constructed which represents the contribution of image cell $j$ to projection pixel $i$. Because the $r-z$ trajectories in the image space are curved (see Fig. 5), the weights $R_{i j}$ are calculated by finely discretizing the viewing line into a set of path elements and allocating the element contributions according to their $(r, z)$ location. The matrix accommodates the physical structure of the divertor and incorporates other weights as appropriate. It is possible to smooth the brightness response matrix in the direction of the fringes to account for the spatial smoothing over the fringes required to isolate the brightness $I_{0}$. In practice this is not necessary because of the binning of the final projection data $688 \times 520 \rightarrow 86 \times 65$ required to reduce the time required to invert the projection image sequence. Because the magnetic configuration is changing dynamically, a new flow-field response matrix must be constructed for each image in sequence. Typically, the number of measurements exceeds the number of reconstructed pixels by a factor of 1.6.

The tomography problem is to solve the linear system $g=R f+d$ where $g$ is the projection, $f$ is the discretized image, and $d$ represents the measurement uncertainties. It is well known that the solution that minimizes the squared residuals $(g-R f)^{T}(g-R f)$ is obtained by solving $R^{T} g=R^{T} R f$. We have implemented an arithmetic simultaneous iterative reconstruction technique ${ }^{14}$ that attempts to find this solution iteratively as follows:

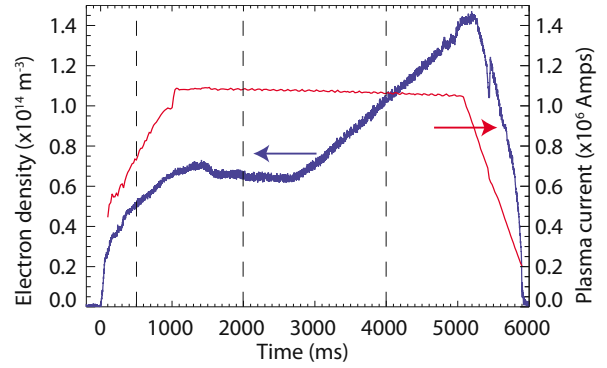

FIG. 6. (Color online) The plasma current and central line-density temporal evolution for discharge no. 141170. The dashed lines correspond to the times selected for analysis of the projection images.

$$
\begin{aligned}
& f^{(0)}=R^{T} g, \\
& f^{(k+1)}=f^{(k)}+\lambda\left[R^{T} g-R^{T} R f^{(k)}\right],
\end{aligned}
$$

where $\lambda$ is an empirically chosen relaxation parameter.

A feature of the iterative approach is that it allows prior information such as positivity and bound constraints to be imposed at each step of the iteration. While unconstrained parallel flow reconstructions are satisfactory, we have correlated adjacent pixels by applying a relaxation parameter image that favors a smooth flow reconstruction as follows:

$$
f_{j}^{(k+1)}=f_{j}^{(k)}+\lambda_{j}^{(k)} \frac{1}{\hat{f}} \sum_{i} R_{i j} \frac{1}{\hat{g}}\left[g_{i}-\sum_{j} R_{i j} f_{j}^{(k)}\right],
$$

where $\hat{f}=\sum_{i} R_{i j}$ and $\hat{g}=\sum_{j} R_{i j}$ are normalizing terms. The relaxation image is given by

$$
\lambda_{j}^{(k)}=\lambda_{0}\left|f_{j}^{(k)} /\left(f_{j}^{(k)} * S\right)\right|,
$$

where $\lambda_{0}$ is a constant relaxation parameter usually set empirically at $\lambda=0.5$ and the convolving function $S$ is here implemented via a three-point Gaussian smoothing kernel. The effect of the relaxation image $\lambda_{j}^{(k)}$ is to reduce the rate at which smooth regions are influenced by successive corrections. The iterations are terminated when the fractional change between iterations is less that 0.001 . The imposition of a smoothness bias is not found to significantly decrease the rate of convergence or to elevate the final residual.

As mentioned previously, uncompensated vignetting of the observed images distorts the reconstructions (a vessel
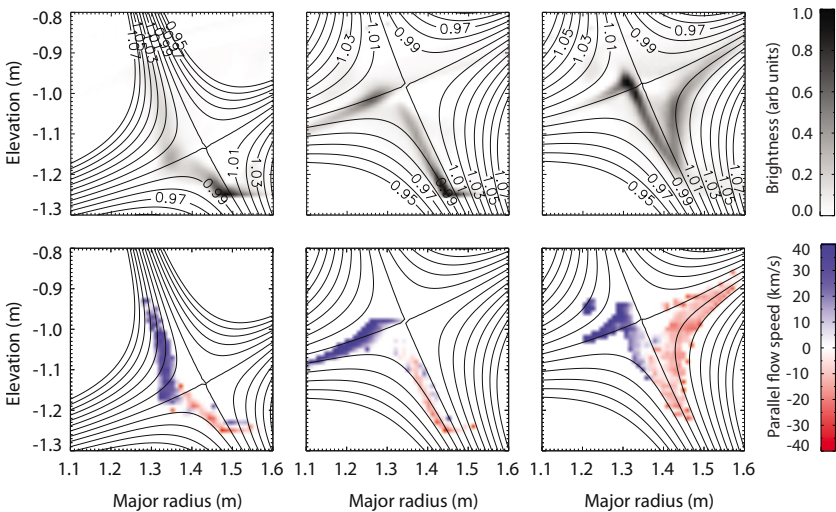

FIG. 7. (Color online) Reconstructions of the emissivity and parallel flow speed at times (L-R) 500, 2000, and $4000 \mathrm{~ms}$. The equilibrium flux surfaces are overplotted for reference. 

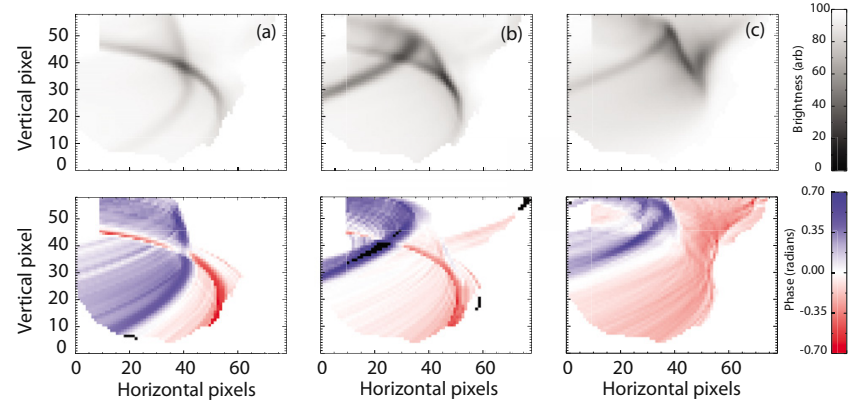

FIG. 8. (Color online) The best fit brightness and phase shift projections corresponding to the measurements shown in Fig. 4.

opening for calibration would have allowed this issue to be addressed). As a first attempt to self-consistently offset the effects of vignetting, we have developed a compensating image formed from an average over an ensemble of brightness projections, of the ratio of the observed projections to their tomographic best fit. This self-consistent compensation is feasible because the projection of an image point at $(r, z)$ produces a crescentlike response that couples large regions of the projection image (these structures are apparent in Fig. 4). We have found empirically that the independent estimates of the vignetting obtained from projections of the diffuse plasma breakdown give a reasonably consistent estimate of the correction, and that its application reduces the reconstruction residual by $\sim 20 \%$ and suppresses some reconstruction artifacts. While the reconstructions shown here utilize this correction, in practice, the differences between reconstructions with and without the vignetting compensation are not major.

\section{EXPERIMENTAL RESULTS AND DISCUSSION}

We consider the tomographic inversions of the projections shown in Fig. 4. The conditions for this discharge number 141170 are $B_{T}=-2.02 \mathrm{~T}, I_{p}=1.1 \mathrm{MA}$ with the electron density ramping from $n_{e} \sim 0.7 \times 10^{14} \mathrm{~m}^{-3}$ to 1.4 $\times 10^{14} \mathrm{~m}^{-3}$ between $t=2700-5200 \mathrm{~ms}$ and with detachment commencing at $t=3200 \mathrm{~ms}$ (see Fig. 6).

Reconstructions of the emissivity and parallel flow speed at times of 500, 2000, and $4000 \mathrm{~ms}$ are shown in Fig. 7. Displacements between the separatrix and regions of maximum brightness suggest a minor inaccuracy in the registration of the camera viewport. The fitted projections are shown in Fig. 8. Residual discrepancies between the projections (Fig. 4) and their best fit can be mostly attributed to the viewport misalignment, image vignetting, and demodulation artifacts. Being a double inversion, the flow reconstructions suffer from uncertainties in the value of the reconstructed emissivity $e(r, z)$ [see Eq. (2)]. While the inversion algorithm has been tested satisfactorily on numerical phantoms, we have not yet undertaken a study of the sensitivity of the flow reconstructions to uncertainties in $e(r, z)$.

Because the poloidal field is in opposite directions for the inboard and outboard divertor legs, we interpret the reversal of the flow direction between the high and low field sides of the X-point as implying plasma flow into the divertors. This behavior, and the reconstructed flow amplitudes, is consistent with earlier observations of near sonic flows in the inboard SOL toward the divertor and into the outboard divertor as the plasma nears detachment. ${ }^{15}$

UEDGE numerical simulations of expected flow speeds in the divertor for different core densities corresponding to times of $4000 \mathrm{~ms}$ (low density case) and $4500 \mathrm{~ms}$ (high density) have been undertaken. The simulations indicate that the SOL and divertor poloidal and radial flows are small $(<10 \%)$ compared with the toroidal component and can be neglected. The simulations at $4000 \mathrm{~ms}$ (see Fig. 9) show the CIII emission peak at the outer leg is located near the divertor plate, detaching at later times $(4500 \mathrm{~ms})$. The simulations also show that the magnitude of the toroidal velocity does not change significantly between 4000 and $4500 \mathrm{~ms}$.

The simulations and measurements exhibit reasonable qualitative agreement. The disagreement in flow amplitudes may simply be attributable to reconstruction inaccuracies as indicated above. However, we observe detachment of the radiating zone at lower densities, and also find that the flow speed in the outer leg increases with increasing density. These structural and temporal trends are experimentally more robust than the inferred flow amplitudes, and indicate that additional systematic studies may be required to resolve the discrepancies between simulation and experiment.

\section{ACKNOWLEDGMENTS}

This work is supported by International Science Linkages established under the Australian Government's innovation statement, "Backing Australia's Ability." This work was also supported in part by the U.S. Department of Energy under Grant Nos. DE-AC52-07NA27344 and DE-FC0204ER54698.
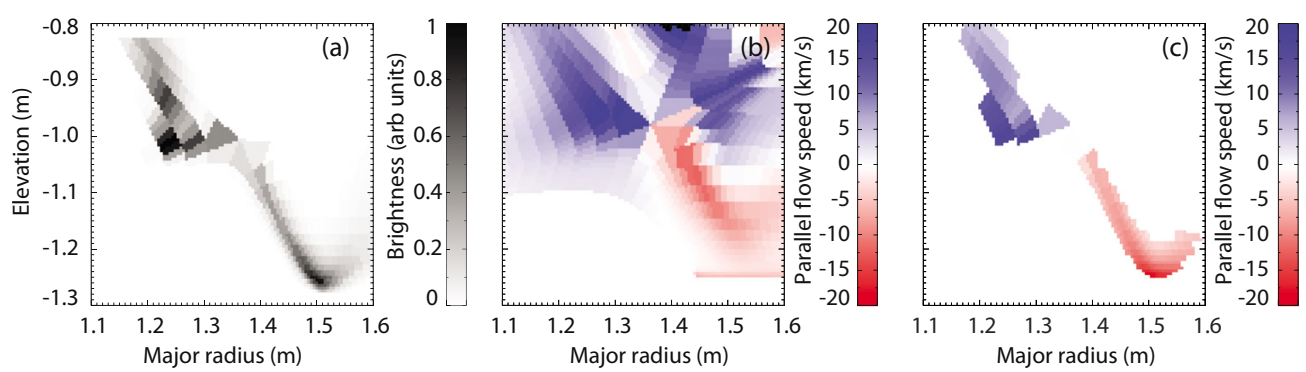

FIG. 9. (Color online) UEDGE simulations of the brightness and toroidal flow fields under conditions corresponding to those at $t=4000 \mathrm{~ms}$ for shot no. 141170 . (a) The local emissivity, (b) the toroidal component of the flow field, and (c) the flow field as seen through an emissivity window masked at the $10 \%$ level. 


\section{APPENDIX: INTERFEROMETER RESPONSE}

We consider a simple polarization interferometer of phase delay offset $\phi_{0}=2 \pi \nu_{0} \tau_{0}$ where $\tau_{0}=L B\left(\nu_{0}\right) / c$ is the associated time delay produced by a uniaxial birefringent plate of thickness $L$ and birefringence $B=n_{E}-n_{O}$ where $n_{E}$ and $n_{O}$ are the wavelength-dependent extraordinary and ordinary wave refractive indices, respectively. When observing quasimonochromatic radiation from an inhomogeneous plasma, the signal at the output port of the interferometer is given by

$$
S_{ \pm}=\frac{I_{0}}{2}\left\{1 \pm \mathfrak{R}\left[\gamma\left(\hat{\phi}_{0} ; \hat{l}\right) \exp \left(\mathrm{i} \phi_{0}\right)\right]\right\},
$$

where $\gamma\left(\hat{\phi}_{0} ; \hat{l}\right)$ is the temporal-coherence of the spectral line, $\hat{l}$ is the unit vector in the direction of view, and the brightness $I_{0}$ is the line integral of the emissivity $e(\boldsymbol{r})$

$$
I_{0}=\int_{L} e(\boldsymbol{r}) d l .
$$

Note that $\gamma$ is a function of the group phase delay $\hat{\phi}_{0}=\kappa \phi_{0}$, where

$$
\kappa=1+\left.\frac{\nu_{0}}{\tau_{0}} \frac{\partial \tau}{\partial \nu}\right|_{\nu_{0}}
$$

accounts for the optical frequency dispersion of the time delay.

For an inhomogeneous plasma in drifting local thermal equilibrium, the temporal coherence is given by ${ }^{12}$

$$
\gamma\left(\hat{\phi}_{0} ; \hat{l}\right)=\frac{1}{I_{0}} \int_{L} e(\boldsymbol{r}) \exp \left(\mathrm{i} \hat{\phi}_{0} \boldsymbol{v}_{D} \cdot \hat{\boldsymbol{l}} / c\right) G_{0}\left(\boldsymbol{r}, \hat{\boldsymbol{\phi}}_{0}\right) d l,
$$

where the complex exponential is due to the Doppler shift of the spectrum and $G_{0}\left(r, \hat{\phi}_{0}\right)$ is the Fourier transform of the Doppler broadened multiplet spectrum. Substituting Eq. (A4) into Eq. (A1) delivers

$$
S_{ \pm}=\frac{I_{0}}{2}\left[1+\zeta_{D} \cos \left(\phi_{0}+\phi_{D}\right)\right],
$$

where $\tan \phi_{D}=\gamma_{i} / \gamma_{r}$ and $\zeta_{D}=|\gamma|$ with $\gamma \equiv \gamma_{r}+i \gamma_{i}$.

For a simple spectral line, $G_{0}$ is the real Gaussian function

$$
G_{0}\left(\boldsymbol{r}, \hat{\phi}_{0}\right)=\exp \left[-T_{S}(\boldsymbol{r}) / T_{C}\right] d l,
$$

where the "characteristic" temperature is fixed by the delay plate properties ${ }^{4}$

$$
k T_{C}=2 m_{S} c^{2} / \hat{\phi}_{0}^{2}
$$

and where $m_{S}$ is the species mass and $T_{S}$ is the species temperature.

We now introduce some approximations appropriate for the conditions of the divertor Doppler measurements. For the chosen $4 \mathrm{~mm}$ thick lithium niobate delay plate, the instrument characteristic temperature $T_{C} \sim 310 \mathrm{eV}$ is significantly larger than the expected range of ion temperatures (10-20 $\mathrm{eV}$ ) allowing the complex quantity $G_{0}$ to be removed from the integral in Eq. (A4). (Experimentally it has been confirmed that the variation in fringe contrast across the interferometric projection is small). The maximum measured phase shifts are also small, of order $0.6 \mathrm{rad}$, so the trigonometric terms are expanded to third order to obtain

$$
\begin{aligned}
\tan \phi_{D}= & \frac{1}{I_{0}} \int_{L} e \widetilde{\phi}_{D} d l-\frac{1}{I_{0}} \int_{L} e\left(\widetilde{\phi}_{D}^{3} / 6\right) d l \\
& +\left(\frac{1}{I_{0}} \int_{L} e \widetilde{\phi}_{D} d l\right)\left[\frac{1}{I_{0}} \int_{L} e\left(\widetilde{\phi}_{D}^{2} / 2\right) d l\right],
\end{aligned}
$$

where $\widetilde{\phi}_{D}(\boldsymbol{r} ; \hat{\boldsymbol{l}})=\hat{\phi}_{0} \boldsymbol{v}_{D} \cdot \hat{\boldsymbol{l}} / c$. For a uniformly drifting homogeneous plasma, the quantities $\phi_{D}$ and $\widetilde{\phi}_{D}$ are equivalent.

In the inhomogeneous case, and when higher order terms are small, we obtain the first order approximation

$$
\phi_{D}=\frac{\hat{\phi}_{0}}{I_{0} c} \int_{L} e(\boldsymbol{r}) \boldsymbol{v}_{D} \cdot d \boldsymbol{l} .
$$

Noting the approximation $\tan \phi_{D} \approx \phi_{D}+\phi_{D}^{3} / 3$, inspection of Eq. (A8) suggests that the higher order terms $\phi_{D}^{3} / 3$ can be largely attributed to the higher order integral terms and therefore that Eq. (A9) will be a very good approximation even when the observed Doppler phase shifts are not small.

${ }^{1}$ R. E. Bell, L. E. Dudek, B. Grek, D. W. Johnson, and R. W. Palladino, Rev. Sci. Instrum. 70, 821 (1999).

${ }^{2}$ G. R. McKee, C. Fenzi, R. J. Fonck, and M. Jakubowski, Rev. Sci. Instrum. 74, 2014 (2003).

${ }^{3}$ M. A. Van Zeeland, J. H. Yu, N. H. Brooks, W. W. Heidbrink, K. H. Burrell, R. J. Groebner, A. W. Hyatt, T. C. Luce, N. Pablant, W. M. Solomon, and M. R. Wade, Plasma Phys. Controlled Fusion 52, 045006 (2010).

${ }^{4}$ J. Howard, C. Michael, F. Glass, and A. Danielsson, Plasma Phys. Controlled Fusion 45, 1143 (2003).

${ }^{5}$ J. Howard et al., "Coherence imaging of flows in the DIII-D divertor," Contrib. Plasma Phys. (submitted).

${ }^{6}$ J. Howard, J. Phys. B 43, 144010 (2010).

${ }^{7}$ M. Francon and S. Mallick, Polarization Interferometers (WileyInterscience, London, 1971).

${ }^{8}$ J. Howard, Plasma Phys. Controlled Fusion 50, 125003 (2008).

${ }^{9}$ J. Howard, A. Diallo, R. Jaspers, and J. Chung, J. Plasma Fusion Res. 5, S1010 (2010).

${ }^{10}$ R. Isler, N. H. Brooks, W. P. West, and G. D. Porter, Phys. Plasmas 6, 1837 (1999).

${ }^{11}$ G. J. Gafert, K. Behringer, D. Coster, C. Dorn, A. Kallenbach, R. Schneider, U. Schumacher, and ASDEX Upgrade Team, J. Nucl. Mater. 266-269, 365 (1999).

${ }^{12}$ J. Howard, F. Glass, and C. Michael, J. Plasma Fusion Res. 2, S1014 (2007).

${ }^{13}$ T. Rognlien, J. Milovich, M. Rensink, and G. Porter, J. Nucl. Mater. 196-198, 347 (1992).

${ }^{14}$ P. Gilbert, J. Theor. Biol. 36, 105 (1972).

${ }^{15}$ M. E. Fenstermacher, J. Boedo, R. C. Isler, A. W. Leonard, G. D. Porter, D. G. Whyte, R. D. Wood, S. L. Allen, N. H. Brooks, R. Colchin, T. E. Evans, D. N. Hill, C. J. Lasnier, R. D. Lehmer, M. A. Mahdavi, R. Maingi, R. A. Moyer, T. W. Petrie, T. D. Rognlien, M. J. Schaffer, R. D. Stambaugh, M. R. Wade, J. G. Watkins, W. P. West, and N. Wolf, Plasma Phys. Controlled Fusion 41, A345 (1999). 